Journal of Computer Science 7 (3): 348-351, 2011

ISSN 1549-3636

(C) 2011 Science Publications

\title{
An Effective Queue Management Scheme for Data Communication
}

\author{
${ }^{1}$ Sheela Thavasi and ${ }^{2}$ Nityanandam Natarajan \\ ${ }^{1}$ Department of IT, Sri Sairam Engineering College, West Tambaram, Chennai \\ ${ }^{2}$ Department of CSE, Sri Sairam Engg. College, Chennai
}

\begin{abstract}
Problem statement: The growth in the needs of Internet applications that transmit massive amount of data has led to the emergence of Effective Queue Management. Approach: Queues were used to smooth spikes in incoming packet rates and to allow the router sufficient time for packet processing. When the waiting time is less a better queue management is ensured thereby resulting in increased flow in the queue. When the incoming packet rate was higher than the router's outgoing packet rate, the queue size was increased, eventually exceeding the available buffer space. With respect to TCP's RED queue management, the problem was that the buffer size was reduced to half and the packets were dropped abruptly. The congestion was detected early and the packets were dropped. The merit may be that congestion was detected early but the adverse effect was loss of packets. Results: The sender had to retransmit causing delay onto the network. This caused adverse effects on the network performance. In proposed system queue was extended whenever the number of packets present in the queue exceeds queue threshold. Packet drops were insignificantly even during network congestion. When the queue was continuously extended and if there was steady inflow of high volume of data, packet drop ratio was insignificant. Conclusions: Thus by the adoption of the proposed work, network throughput rose considerably thereby enabling effective data transfer. So it exhibits high bandwidth utilization, good throughput and processing time.
\end{abstract}

Key words: Adaptive Queue Control (AQC), data rate, Internet Protocol (IP), Transmission Control Protocol (TCP), queue management, data communication, network congestion, bandwidth utilization, increases gradually, proposed mechanism

\section{INTRODUCTION}

The Internet carries certain critical data and information which needs to be delivered to the receiver at all cost (Huang et al., 2008). The primary factor hindering the flow of traffic is the network congestion. Also another issue in data communication is the speed of data transmission. $56 \mathrm{kbps}$ was the fastest speed in the late 1960s but today speeds of 112 Mbps are achieved for household usage. The need to acquire vast amount of information within a short duration requires high speed Internet. Fig. 1 describes the evolution of internet with respect to link capacity which was initially in bits per second and over the period of time the usage changed from bps to Kbps, Kbps to Mbps, Mbps to Gbps and of late, from Gbps to Tbps. The need for various applications to transmit massive amount of data along with seamless flow within a short duration has led to the emergence of high-speed network.

For the continuous flow of data, the queue management scheme is important. In this regard Packets are dropped from the queue where the queue size exceeds the queue threshold value. So the Packet drop ratio becomes high leading to the reduction in throughput. It is determined that the above mentioned issues lead to poor utilization, maximum delay and eventually minimum throughput. Implementation of queue management technique which extends the queue whenever the number of packets present in the queue exceeds queue threshold (Ostadabbas and Haeri, 2007) Packet drops are insignificant even during network congestion. Overall the primary motivation of our research is to achieve better reliability, scalability, maximum throughput, minimum delay and high utilization for effective data transfer.

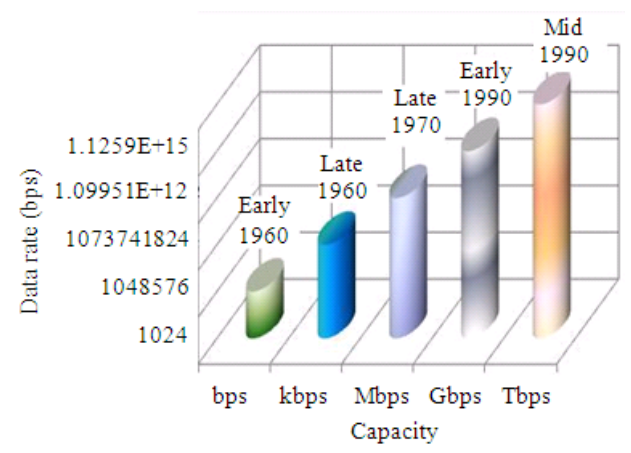

Fig. 1: Evolution of bandwidth capacity

Corresponding Author: T. Sheela, Department of IT, Sri Sairam Engineering College, West Tambaram, Chennai 


\section{MATERIALS AND METHODS}

Queue management: In case of the traditional TCP protocol, the RED queue management technique is adopted (Rawajbeh et al., 2010) RED stands for Random Early Detection. Any protocol that runs on top of Internet Protocol (IP), such as Transmission Control Protocol (TCP), can detect packet drops and interpret them as indications of congestion in the network. In particular, a TCP sender will react to these packet drops by reducing its sending rate. This slower sending rate translates into a decrease in the incoming packet rate at the router, which effectively allows the router to clear up its queue. Queues are used to smooth spikes in incoming packet rates and to allow the router sufficient time for packet transmission. When the incoming packet rate is higher than the router's outgoing packet rate, the queue size will increase, eventually exceeding the available buffer space. When the buffer is full, some packets will have to be dropped. But with respect to TCP's RED queue management, the problem is the buffer size is reduced to half and the packets are dropped abruptly. The congestion is detected early and the packets are dropped (Aziz et al., 2011; Reddy and Ahammed, 2008). The merit may be that congestion is detected early but the adverse effect is loss of packets. As a result the sender has to retransmit causing delay onto the network. This causes adverse effects on the network performance (Ostadabbas and Haeri, 2007).

Network traffic prediction is important for network control and bandwidth calculation. Describes the threshold crossing analysis for high speed network traffic (Huang et al., 2008) uses the closed queuing network in heavy network traffic. Network buffers (packet buffers) are used in almost all types of network switches and routers to buffer incoming flow of packets. Speed and capacity of packet buffers must be increased as the line rate of the network communication links increases. An Internet router typically maintains a set of queues that hold packets meant for sending to the appropriate destination. Historically, such queues use a drop tail discipline: a packet is put onto the queue if the queue is shorter than its maximum size (measured in packets or in bytes) and dropped otherwise (Ostadabbas and Haeri, 2007). The packets from the senders are accommodated in individual queue and forwarded to the out queue on round robin basis. If the congestion is detected the router enters into the wait mode and the senders have to reduce their sending rate and if they fail to comply with their rate reduction then such nodes are treated as misbehaving source and all the packets are dropped from queues of the corresponding senders .If the senders are designated as misbehaving nodes, then the drop ratio increases and automatically the throughput is minimized. Active queue usually drop or mark packets before the queue is full. Typically, they operate by maintaining one or more drop/mark probabilities (Reddy and Reddy, 2009). A self tuning adaptive control algorithm called Adaptive Queue Control (AQC) is the approach used for active queue management in TCP network. The queue size is fixed and it does not vary under any circumstances. Based on the Residing Time and Waiting Time, Processing Time is calculated. The Packets occupy Buffers and are not dropped abruptly. The queue length is calculated based on the link Capacity. In the case of an over estimation of the capacity the stabilization queue length will be positive, otherwise it will be negative (Nabeshima and Yata, 2005; Hassan et al., 2009).

\section{Queue model:}

Load on the network: It is based on the flow per second in the network.

For $n$ flows, Load is given by $\sum \lambda_{\mathrm{i}} \mathrm{i}=1$ to $\mathrm{n}$ where $\mathrm{n}$ is the number of flows.

$\lambda$ - Number of packets

$\mathrm{T}_{\mathrm{s}}($ ServiceTime $)=$ Packet Size/Bandwidth Capacity $(1)$

The time required to service a single packet on the network:

$\rho($ Utilization $)=\lambda * T_{\mathrm{s}}$

The amount of bandwidth and resources utilized:

Queue Size $(r)=\rho /(1-\rho)$

The queue size allocated for transfer:

Resident Time $\left(\mathrm{T}_{\mathrm{r}}\right)=\mathrm{T}_{\mathrm{s}} /(1-\rho)$

The Time the packet spends in the queue:

Waiting Time $\left(\mathrm{T}_{\mathrm{w}}\right)=\rho \mathrm{T}_{\mathrm{s}} /(1-\rho)$

Mean queue waiting time:

Processing Time $\left(T_{p}\right)=T_{s}+T_{w}$

The Net Time required to render a response:

Queue threshold (Q) $=95.35 \%$ of $\mathrm{r}$

\section{RESULTS AND DISCUSSION}

Queue delay: In the proposed mechanism only minimum delay is experienced even under severe load condition. The delay is calculated based on the Eq. 5 . 
J. Computer Sci., 7 (3): 348-351, 2011

Table 1: Queue parameters

\begin{tabular}{llclll}
\hline Data rate (Mbps) & $\begin{array}{l}\text { Number of } \\
\text { Packets }\end{array}$ & $\begin{array}{l}\text { Queue Size } \mathrm{r} \\
\text { (packets) }\end{array}$ & $\begin{array}{l}\text { Resident time } \\
\mathrm{T}_{\mathrm{r}}(\mathrm{sec})\end{array}$ & $\begin{array}{l}\text { Waiting time } \\
\mathrm{T}_{\mathrm{w}}(\mathrm{sec})\end{array}$ & $\begin{array}{l}\text { Processing time } \\
\mathrm{Tp}=\mathrm{T}_{\mathrm{s}}+\mathrm{T}_{\mathrm{w}}(\mathrm{sec})\end{array}$ \\
\hline 7 & 612 & 0.079 & 0.000120 & $9.5 \times 10^{-6}$ & 0.0001200 \\
18 & 1572 & 0.232 & 0.000140 & $2.7 \times 10^{-5}$ & 0.0001400 \\
25 & 2184 & 0.350 & 0.000162 & $4.2 \times 10^{-5}$ & 0.0001626 \\
36 & 3145 & 0.606 & 0.000190 & $7.2 \times 10^{-5}$ & 0.0001900 \\
40 & 3495 & 0.720 & 0.000206 & $8.6 \times 10^{-5}$ & 0.0002060 \\
53 & 4631 & 1.250 & 0.000270 & $1.5 \times 10^{-4}$ & 0.0002700 \\
62 & 5417 & 1.850 & 0.000340 & 0.000220 & 0.0003400 \\
75 & 6500 & 3.500 & 0.000545 & 0.000425 & 0.0005450 \\
84 & 7340 & 7.380 & 0.000880 & 0.000880 & 0.0010000 \\
95 & 8301 & 258.000 & 0.031100 & 0.031000 & 0.0311200 \\
95.3 & 8327 & 1249.000 & 0.150000 & 0.149880 & 0.1500000 \\
95.31 & 8328 & 1659.000 & 0.196720 & 0.196600 & 0.1967200 \\
\hline
\end{tabular}

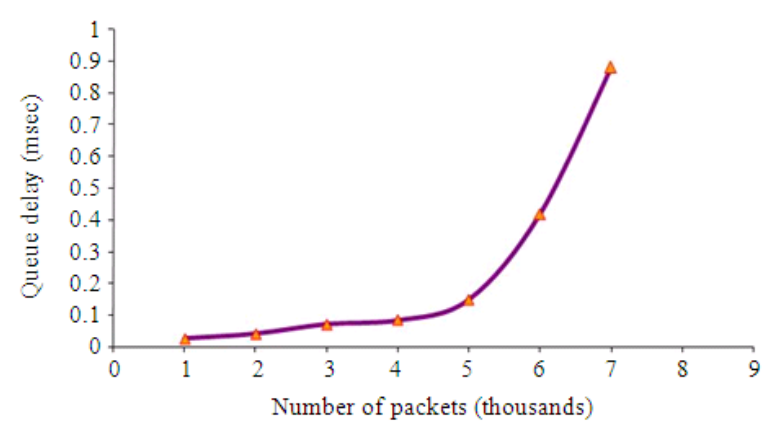

Fig. 2: Number of packets (Thousands) Vs queue delay (msec)

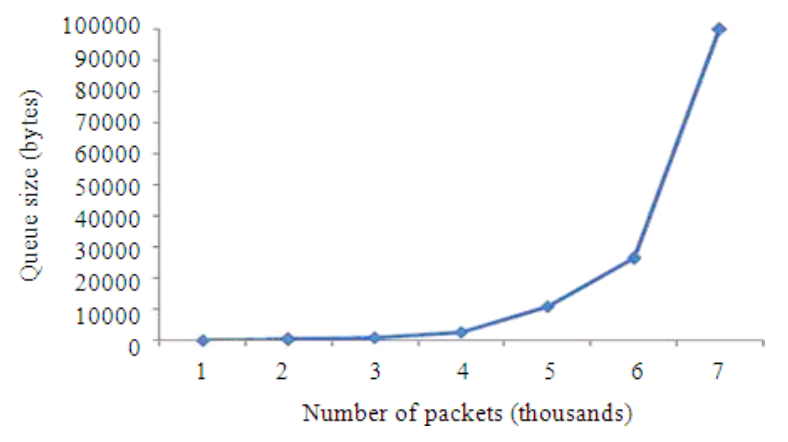

Fig. 3: Number of packets Vs queue size

The queuing delay is based on the utilization $(\rho)$ and queue size (r). The delay time is from $9.5 \times 10^{-6} \mathrm{sec}$, which is the delay with respect to the minimum load, to $0.14 \mathrm{sec}$ for more than 8000 packets and it is represented in the Fig. 2. Even though the load increases in the network, only minimum delay is experienced at the time of severe congestion. Whenever network congestion occurs and for the subsequent high data rates the queuing delay increases gradually. But even at severe congestion, the queuing delay is around $1 \mathrm{~ms}$, as shown in the Table 1, which is $10-15 \mathrm{sec}$ lesser than the delay experienced during severe congestion in the existing mechanism. The processing time is calculated based on queue waiting time (Queuing delay) and service time. The service time $T_{S}$ is calculated based on Eq. 1 and the value is $0.00012 \mathrm{sec}$ for a bandwidth of 100 Mbps.

Queue size: The following Fig. 3 reflects the data rate and corresponding queue size. The number of packets that reside in the queue at low data rates is only around one packet. The service time is $0.00012 \mathrm{sec}$ and 0.000012 at the bandwidth of $100 \mathrm{Mbps}$ and $1 \mathrm{Gbps}$ respectively. In the proposed mechanism, when the network congestion occurs queue size is determined using the effective queue management mechanism which falls under two categories depending upon whether it is medium or severe network congestion. When medium congestion occurs, the queue size is increased by $10 \%$ and when severe congestion occurs, the queue size is extended by $20 \%$ to accommodate the additional packets. As the queue is continually extended and if there is steady inflow of high volume of data, packet drop ratio is insignificant.

Processing time: Table 1 show the various queue parameters and also the processing time corresponding to various data rates. The processing time is an insignificant value at lower data rates and the processing time increases steadily as the data rate increases steadily the processing time also increases gradually. For example, at $7 \mathrm{Mbps}$ data rate the processing time is $0.00014 \mathrm{sec}$ and it increases to 0.00034 for data rate of $62 \mathrm{Mbps}$ and further rises to 0.000545 for data rate of 75 Mbps. The following Fig. 4 shows the processing time at the data rate from 25 Mbps to 820 Mbps with the bandwidth capacity of 1 Gbps. The processing time required to transfer the data rate of $820 \mathrm{Mbps}$ is $8 \times 10^{-5}$. 


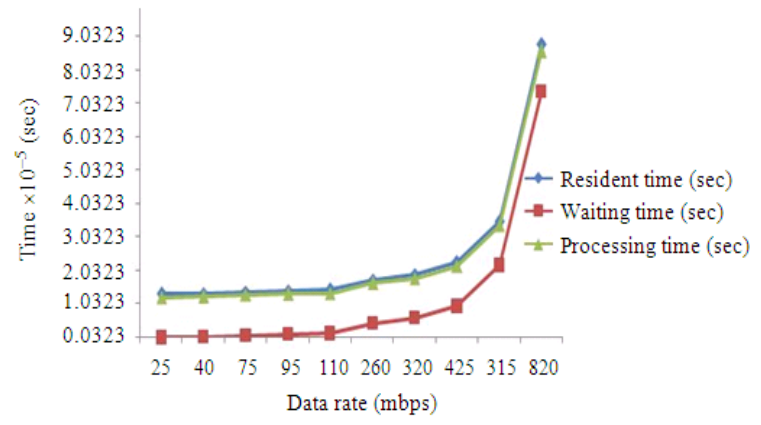

Fig. 4: Data rate Vs time (1 Gbps BW)

\section{CONCLUSION}

Reliability and Effectiveness are the essentials which should be satisfied by High Speed Networks. The Proposed Work upgrades the functionality of the Existing protocols. By adopting multiple congestion control techniques effectiveness in transmission and reception is achieved. In the Proposed Work, the algorithms for queue Management achieves 96\% efficiency. The window size is altered drastically in the existing system based on the time and packet flow and results in poor bandwidth utilization. But in the proposed work, the procedure is based on the bandwidth and feedback, where the input packets are regulated by altering the source node and thereby reducing the window size in unit step. So the sender can restrict the flow and can avoid congestion which results in effective bandwidth utilization. Also the feedback mechanism in the proposed work enables detection of congestion effectively. This mechanism is highly useful, particularly in large Networks which constitute about 1000 senders who transfer continuously at varying data rates. The proposed work offers a better mechanism for seamless flow of voluminous data, better throughput, minimum delay and high bandwidth utilization.

\section{REFFERENCES}

Aziz, A.A., M.Y.M. Saman and M.P. Hamzah, 2011. Using metadata analysis and base analysis techniques in data qualities framework for data warehouses. Am. J. Econ. Bus. Admin., 3: 112119. DOI: 10.3844/ajebasp.2011.112.119

Hassan, R., J. Irvine and I. Glove, 2009. Design and analysis of virtual bus transport using synchronous digital hierarchy/synchronous optical networking. J. Comput. Sci., 4: 1003-1011. DOI: 10.3844/jcssp.2008.1003.1011

Huang, X., F. Ren, G. Yang, Y. Wu and W. Zhen et al., 2008. End-to-end congestion control for high speed networks based on population ecology models. Proceedings of the 28th International Conference on Distributed Computing Systems, June 17-20, Beijing, pp: $353-360 . \quad$ DOI: 10.1109/ICDCS.2008.26

Nabeshima, M. and K. Yata, 2005. Performance improvement of active queue management with per-flow scheduling. IEEE Proc. Commun., 152: 797-803. DOI: 10.1049/ip-com:20045217

Ostadabbas, S. and M. Haeri, 2007. Adaptive congestion estimation and control in active queue management. Proceedings of the International Conference on Control, Automation and System, Oct. 17-20, Seoul, pp: 2850-2853. DOI: 10.1109/ICCAS.2007.4406855

Rawajbeh, M.A., I. Mansi and M. Hussien, 2010. Algorithm development for measurement and prediction the traffic in broad band integrated service networks. J. Comput. Sci., 6: 1164-1169. DOI: $10.3844 /$ jcssp.2010.1164.1169

Reddy, S. and L.C. Reddy, 2009. A modified model for efficient explicit congestion reduction in high traffic high speed networks through automated rate controlling. Proceedings of the IEEE International Advance Computing Conference, Mar. 6-7, Patiala, pp: 664-669. DOI: 10.1109/IADCC.2009.4809091

Reddy, T.B. and A. Ahammed, 2008. Performance comparison of active queue management techniques. J. Comput. Sci., 4: 1020-1023. DOI: 10.3844/jcssp.2008.1020.1023 